OPEN ACCESS

Edited by:

Yong Chen,

Duke University, United States

Reviewed by:

Zilong Wang,

Southern University of Science and Technology, China

Changyu Jiang,

Duke University, United States

*Correspondence: Zhi-Yong Tan

zt2@iupui.edu

${ }^{\dagger}$ These authors have contributed equally to this work

Specialty section:

This article was submitted to Pain Mechanisms and Modulators, a section of the journal Frontiers in Molecular Neuroscience

Received: 04 April 2021 Accepted: 10 May 2021

Published: 04 June 2021

Citation:

Wu B, Su X, Zhang W, Zhang $Y$-H, Feng $X$, Ji Y-H and Tan Z-Y (2021) Oxaliplatin Depolarizes the IB4-

Dorsal Root Ganglion Neurons to Drive the Development of Neuropathic Pain Through TRPM8

in Mice.

Front. Mol. Neurosci. 14:690858. doi: 10.3389/fnmol.2021.690858

\section{Oxaliplatin Depolarizes the IB4- Dorsal Root Ganglion Neurons to Drive the Development of Neuropathic Pain Through TRPM8 in Mice}

\author{
Bin Wu ${ }^{1,2+}$, Xiaolin Su ${ }^{2,3+}$, Wentong Zhang ${ }^{2}$, Yi-Hong Zhang ${ }^{2}$, Xinghua Feng 4 , \\ Yong-Hua Ji5 and Zhi-Yong Tan ${ }^{2 *}$
}

1 Institute of Special Environment Medicine, Nantong University, Nantong, China, ${ }^{2}$ Department of Pharmacology and Toxicology, Stark Neurosciences Research Institute, Indiana University School of Medicine, Indianapolis, IN, United States, ${ }^{3}$ Department of Biochemistry and Molecular Biology, Indiana University School of Medicine, Indianapolis, IN, United States, ${ }^{4}$ Collaborative Innovation Center of Yangtze River Delta Region Green Pharmaceuticals, College of Pharmaceutical Sciences, Zhejiang University of Technology, Hangzhou, China, ${ }^{5}$ Laboratory of Neuropharmacology and Neurotoxicology, Shanghai University, Shanghai, China

Use of chemotherapy drug oxaliplatin is associated with painful peripheral neuropathy that is exacerbated by cold. Remodeling of ion channels including TRP channels in dorsal root ganglion (DRG) neurons contribute to the sensory hypersensitivity following oxaliplatin treatment in animal models. However, it has not been studied if TRP channels and membrane depolarization of DRG neurons serve as the initial ionic/membrane drives (such as within an hour) that contribute to the development of oxaliplatin-induced neuropathic pain. In the current study, we studied in mice (1) in vitro acute effects of oxaliplatin on the membrane excitability of $\mathrm{IB}^{+}{ }^{+}$and $\mathrm{IB} 4^{-}$ subpopulations of DRG neurons using a perforated patch clamping, (2) the preventative effects of a membrane-hyperpolarizing drug retigabine on oxaliplatin-induced sensory hypersensitivity, and (3) the preventative effects of TRP channel antagonists on the oxaliplatin-induced membrane hyperexcitability and sensory hypersensitivity. We found (1) IB4 ${ }^{+}$and IB4 ${ }^{-}$subpopulations of small DRG neurons displayed previously undiscovered, substantially different membrane excitability, (2) oxaliplatin selectively depolarized IB4- DRG neurons, (3) pretreatment of retigabine largely prevented oxaliplatin-induced sensory hypersensitivity, (4) antagonists of TRPA1 and TRPM8 channels prevented oxaliplatin-induced membrane depolarization, and (5) the antagonist of TRPM8 largely prevented oxaliplatin-induced sensory hypersensitivity. These results suggest that oxaliplatin depolarizes IB4- neurons through TRPM8 channels to drive the development of neuropathic pain and targeting the initial drives of TRPM8 and/or membrane depolarization may prevent oxaliplatin-induce neuropathic pain.

Keywords: oxaliplatin, neuropathic pain, dorsal root ganglion, IB4, initial drive, membrane depolarization, TRPM8 


\section{INTRODUCTION}

The third-generation platinum drug oxaliplatin is the most common used drug for locally advanced and metastatic cancer of the colon, rectum, or pancreas. Compared to classic platinum agents, such as cisplatin, oxaliplatin has lower hematotoxicity and gastrointestinal toxicity (de Gramont et al., 2000). However, unlike other platinum agents which cause the chronic neuropathy, oxaliplatin specifically induced acute painful chemotherapy-induced peripheral neuropathy (CIPN) during or within hours after its first infusion in almost $90 \%$ patients (Leonard et al., 2005; Gebremedhn et al., 2018). The acute pain may become increasingly severe in the subsequent cycles and can become chronic that lasts for months and years (Beijers et al., 2014; Briani et al., 2014). The oxaliplatin-induced CIPN is evoked or exacerbated by cold, with symptoms including throat discomfort, paresthesia and dysesthesia of the hands, feet, and perioral region; these symptoms often lead to reduction or discontinued use of oxaliplatin treatment (Argyriou et al., 2010; Kerckhove et al., 2017).

The mechanisms of oxaliplatin-induced CIPN include DNA damage, mitochondrial dysfunction, calcium chelation, reactive oxygen species (ROS) production, and ion channel remodeling in dorsal root ganglion (DRG) neurons (Joseph et al., 2008; Descoeur et al., 2011; Boyette-Davis et al., 2015; ViatchenkoKarpinski et al., 2018; Rovini, 2019; Trecarichi and Flatters, 2019). For instance, transient receptor potential (TRP) channels expressed on DRG neurons such as TRPV1, TRPA1, and TRPM8 have been studied following the treatment of oxaliplatin (Chukyo et al., 2018). Several studies have shown that the mRNA expression level of TRPA1 and TRPM8, but not TRPV1 were upregulated within several days after oxaliplatin treatment (Gauchan et al., 2009; Ta et al., 2010; Yamamoto et al., 2015; Nakagawa and Kaneko, 2017). More importantly, mechanical allodynia and cold hypersensitivity induced by single or repeated administration of oxaliplatin was abolished by pharmacological inhibition or a gene deficiency of TRPA1 or TRPM8 (Gauchan et al., 2009; Nassini et al., 2011). These findings suggest that TRPA1 and/or TRPM8 channels play important role in the maintaining of oxaliplatin-induced neuropathic pain several days following oxaliplatin treatment. On the other hand, it has not been studied if TRP channels and membrane depolarization of DRG neurons serve as the initial ionic/membrane drives (such as within an hour) that contribute to the development of oxaliplatin-induced neuropathic pain. In the current study, we investigated the role of TRP channels and membrane depolarization in the initial pain-driving process following oxaliplatin treatment using in vitro and in vivo mouse models. We characterized the membrane excitability of $\mathrm{IB}_{4}^{+}$and IB4 ${ }^{-}$ subpopulations of small DRG neurons, examined the responses of $\mathrm{IB}^{+}$and $\mathrm{IB}^{-}$DRG neurons to acute oxaliplatin, and studied the effects of antagonists of membrane depolarization and subtypes of TRP channels on the membrane hyperexcitability and/or pain behaviors induced by oxaliplatin. We found that $\mathrm{IB}^{+}$and $\mathrm{IB}^{-}$subpopulations of small DRG neurons displayed large differences in membrane excitability and that oxaliplatin selectively depolarized $\mathrm{IB}^{-}$neurons. Antagonists of TRPA1 or TRPM8 prevented oxaliplatin-induced membrane depolarization and that targeting membrane depolarization or TRPM8 prevented oxaliplatin-induced neuropathic pain behaviors. There results suggest that TRPM8 and membrane depolarization in $\mathrm{IB}^{-}$neurons might serve as the initial ionic/membrane drives that contribute to the development of oxaliplatin-induced neuropathic pain.

\section{MATERIALS AND METHODS}

\section{Animals}

Male C57BL/6 mice at the age of 8 weeks (Jackson Laboratory, Bar Harbor, ME, United States) were used for cell culture and behavior experiments. Mice were housed five or less per cage at a temperature-controlled room $\left(22 \pm 0.5^{\circ} \mathrm{C}, 12 \mathrm{~h} / 12 \mathrm{~h}\right.$ light/dark cycle) and were with free access to water and pellet diet. All experimental protocols were approved by the Institutional Animal Care and Use Committees of the Indiana University School of Medicine, Indianapolis, IN, United States. All procedures were conducted in accordance with the Guide for Care and Use of Laboratory Animals published by the National Institutes of Health and the ethical guidelines established by the International Association for the Study of Pain.

\section{Drugs and Their Administration}

Oxaliplatin and Capsazepine were purchased from SigmaAldrich (St. Louis, MO, United States). Retigabine was purchased from Alomone Labs (Jerusalem, Israel), A-967079 was purchased from MedChemExpress (Monmouth Junction, NJ, United States), and TC-I was purchased from Tocris, BioTechne Corporation (Minneapolis, MN, United States). For electrophysiology, stock solutions were made in distilled water (oxaliplatin, $10 \mathrm{mM}$ ) or DMSO (A-967079, capsazepine, and TC-I). The stock solutions were diluted by bath solution to reach the final concentration of chemicals including oxaliplatin $(50 \mu \mathrm{M}), \mathrm{A}-967079(1 \mu \mathrm{M})$, capsazepine $(10 \mu \mathrm{M})$ and TC-1 $(10 \mathrm{nM})$. For chemicals diluted from DMSO stocks, the final solution contains $0.1 \%$ DMSO. All these drugs were pre-treated in the bath solution for 15-60 min before electrophysiological recordings. For behavior test, stock solutions of oxaliplatin in distilled water $(5 \mathrm{mg} / \mathrm{ml})$, were further diluted in $5 \%$ glucose to give the final concentration of $1.25 \mathrm{mg} / \mathrm{ml}$. Retigabine, A-967079, TC-1 were dissolved in DMSO and further diluted in saline (to give $2 \% \mathrm{DMSO}$ ) for different final concentrations. A single intraperitoneal (i.p.) injection of oxaliplatin $(5 \mathrm{mg} / \mathrm{kg})$ was conducted and retigabine $(10 \mathrm{mg} / \mathrm{kg}$, i.p.), TC-I $(10 \mathrm{mg} / \mathrm{kg}$, i.p.) and A-967079 (100 mg/kg, p.o.) were administrated daily for 5 days from 2 days before to 2 days after the injection of oxaliplatin. Chemicals without specified sources were from Sigma-Aldrich (St. Louis, MO, United States).

\section{Behavior Test}

All the testing was carried out in accordance with the approved guidelines. All behavioral measurements were performed in conscious, unrestrained, and age-matched adult male mice. 


\section{Mechanical Nociceptor Assay}

As described previously, the von Frey assay of "simplified updown" method was used to assess mechanical sensitivity (Su et al., 2019). Mice were placed in Plexiglas cubicle containers on a metal mesh wire platform to allow access to the plantar hindpaw. A set of eight calibrated von Frey filaments ranging from 0.008 to $6 \mathrm{~g}$ (North Coast Medical, Morgan Hill, CA, United States) were applied alternately to the plantar surface of each hindpaw until they bent. The duration of each stimulus was approximately $1 \mathrm{~s}$ and nociceptive behaviors included retraction/lifting, rapid shaking, and/or licking of the hindpaw.

\section{Heat Nociceptor Assay}

The thermal sensitivity was assessed by applying infrared heating to the plantar surface of hindpaw and the response latency was read from an automated device (IITC model 400, Woodland Hills, CA, United States), as described previously (Zhou et al., 2019). Each hindpaw was tested five times with 5 min interval, and the withdrawal latency was averaged. To avoid tissue damage by prolonged thermal stimuli, cut off latency was set as $20 \mathrm{~s}$.

\section{Cold Nociceptor Assay}

The acetone test was performed as previously described (Su et al., 2019). Mice were placed to the same setting described above for the von Frey test. Fifty microliter of acetone was applied to the center of the ventral side of the hindpaw and responses were observed. In the first $20 \mathrm{~s}$ following acetone application, if the mouse did not withdraw, flick or stamp of the paw then 0 points were recorded for the trial. However, if within this $20 \mathrm{~s}$ period the animal responded to acetone, then the animal's response was assessed for an additional $20 \mathrm{~s}$. Responses to acetone were graded according to the following 4-point scale: 0 , no response; 1 , quick withdrawal, flick or stamp of the paw; 2 , prolonged withdrawal or repeated flicking of the paw; 3 , repeated flicking of the paw with licking directed at the paw. Acetone test was applied alternately three times to each paw and the responses scored categorically.

\section{Rotarod Test}

The rotarod test was conducted according to the previous publication (Liu et al., 2014). The animals had two sessions of training tests before the first scheduled test. At each training or scheduled test, three levels of rotating speed were used: $1-18 \mathrm{rpm}$, 3-30 rpm, and 4-40 rpm. The time for the animal to stay on the rod from the beginning of rod-rotation to the falling of the animal to the ground was recorded. A cut-off time of $120 \mathrm{~s}$ was used.

\section{Cell Culture}

Dorsal root ganglion neurons were dissociated and prepared from adult mice using a similar protocol as previously described (Su et al., 2019). Briefly, mice were sacrificed by exposure to $\mathrm{CO}_{2}$ and decapitated. DRG were rapidly removed and placed in Puck's solution containing digesting enzymes. The DRGs were digested with Liberase TM (0.35 U/ml; SigmaAldrich, St. Louis, MO, United States) for 35-40 min before another $10 \mathrm{~min}$ with Liberase TL $(0.25 \mathrm{U} / \mathrm{ml}$; Sigma-Aldrich, St. Louis, MO, United States) and papain (30 U/ml, Worthington
Biochemical) at $37^{\circ} \mathrm{C}$. The ganglia were then triturated with firepolished Pasteur pipettes. The dispersed cells were resuspended in F12 (Thermo Fisher Scientific, Waltham, MA, United States) medium supplemented with 10\% FBS (Thermo Fisher Scientific, Waltham, MA, United States) and 1\% penicillin/streptomycin (MediaTech, Inc., Manassas, VA, United States) and plated on coverslips coated with polyornithine (Neuvitro Corporation, Vancouver, WA, United States) and laminin. Cell cultures were maintained in regular $95 \%$ air and $5 \% \mathrm{CO}_{2}$ at $37^{\circ} \mathrm{C}$ in an incubator.

\section{Electrophysiological Recordings}

Dorsal root ganglion neurons were recording 16-24 h after dissociation as described previously ( $\mathrm{Su}$ et al., 2019; Wu et al., 2019). Small diameter DRG neurons $(<25 \mu \mathrm{m})$ were chosen for whole-cell patch clamp recording in the currentclamp mode at room temperature. A perforated wholecell patch clamp recording was conducted by including $240 \mu \mathrm{g} / \mathrm{mL}$ Amphotericin B in the pipette solution (Tan et al., 2010). The bath solution consisted of $140 \mathrm{mM}$ $\mathrm{NaCl}, 3 \mathrm{mM} \mathrm{KCl}, 2 \mathrm{mM} \mathrm{MgCl} 2,2 \mathrm{mM} \mathrm{CaCl}$, $10 \mathrm{mM}$ HEPES, pH 7.3. DRG neurons were recorded with firepolished, borosilicate glass patch pipettes (5-8 M $\Omega$ ), which were pulled from borosilicate glass capillaries (Harvard Apparatus, Holliston, MA, United States) using a Sutter P-97 puller (Sutter Instrument, Novato, CA, United States). The pipette solution contained $30 \mathrm{mM} \mathrm{KCl}, 110 \mathrm{mM}$ potassium gluconate, $0.5 \mathrm{mM}$ EGTA, $5 \mathrm{mM}$ HEPES, and $3 \mathrm{mM}$ Mg-ATP, $\mathrm{pH}$ 7.3. Data were acquired using Axopatch 200B patch-clamp amplifier (Molecular Devices Corporation, Sunnyvale, CA, United States) driven by a personal computer in conjunction with an A/D and D/A board (DigiData 1320 A series interface, Molecular Devices Corporation). The action potential (AP) was evoked by depolarizing current steps with long or short time durations $(1,000 \mathrm{~ms}$ or $5 \mathrm{~ms})$. The long current steps were used to test AP rheobases and AP numbers. The short current steps were used to trigger single action potential that was used to calculate the shape properties of APs. Signals were low-pass filtered at $5 \mathrm{kHz}$, sampled at $20 \mathrm{kHz}$ and analyzed offline. To avoid the confounding effects of IB4 staining on the forming of Giga-seal for patch clamp recording and potentially on the electrophysiological properties of DRG neuron, DRG neurons were stained by IB4-FITC (5 $\mu \mathrm{g} / \mathrm{ml}$, incubated for $10 \mathrm{~min}$ ) immediately after recording to distinguish $\mathrm{IB}^{+}$and $\mathrm{IB}^{-}$neurons (Dirajlal et al., 2003; Vilceanu et al., 2010).

\section{Quantification and Statistics}

GraphPad Prism v5.0 was used for statistical analyses. All the results were presented as mean \pm SEM. The behavioral data were analyzed by two-way repeated measures (RM) ANOVA followed by Bonferroni test. The electrophysical recording data were analyzed by Student's $t$-test or two-way RM ANOVA followed by Bonferroni test. For all experiments, $P<0.05$ was considered to be significant $\left({ }^{*} P<0.05,{ }^{* *} P<0.01\right.$, and $\left.{ }^{* * *} P<0.001\right)$. 


\section{RESULTS}

\section{IB4 ${ }^{+}$and IB4- Neurons Showed Substantially Different Electrophysiological Properties}

$\mathrm{IB}^{+}{ }^{+}$and IB4 ${ }^{-}$neurons are two major cell types of small diameter $(<25 \mu \mathrm{m})$ DRG neurons, most of which are nociceptive. To begin with the study, we compared the electrophysiological properties of small $\mathrm{IB}^{+}(n=21)$ and $\mathrm{IB}^{-}(n=38)$ DRG neurons using a perforated current-clamp whole-cell recording (Figure 1A). As shown in Figure 1, the membrane capacitance (Cm, Figure 1B) and membrane resistance (Rm, Figure 1C) were similar between $\mathrm{IB}_{4}^{+}$and $\mathrm{IB}^{-}$neurons. Compared to $\mathrm{IB}^{-}{ }^{-}$neurons, the resting membrane potentials in $\mathrm{IB} 4^{+}$neurons were significantly depolarized and the rheobase currents were lower (Figures 1D-F). Although the number of action potentials, triggered by current injections from 20 to $100 \mathrm{pA}$ were not significantly different (Figure 1G), a suprathreshold current injection (at $3 \times$ rheobase) elicited significantly more action potentials in $\mathrm{IB}^{-}$neurons than those in $\mathrm{IB}^{+}{ }^{+}$ones (Figure $\mathbf{1 H}$ ).

We further compared the properties of single action potentials elicited in $\mathrm{IB}^{+}(n=17)$ and IB4 ${ }^{-}(n=24)$ neurons (Figure 1I). Although the amplitude (Figure 1J), duration (Figure 1K), the maximal rising slope $\left(68.8 \pm 4.3 \mathrm{mV} / \mathrm{ms}\right.$ in $\mathrm{IB}^{+}{ }^{+}$cells and $70.9 \pm 4.0 \mathrm{mV} / \mathrm{ms}$ in IB $^{-}$cells) or decaying slope of action potentials $\left(-55.7 \pm 3.3 \mathrm{mV} / \mathrm{ms}\right.$ in $\mathrm{IB}^{+}$cells and $-64.0 \pm 2.9 \mathrm{mV} / \mathrm{ms}$ in IB4${ }^{-}$cells) were not significantly different between these two cell subtypes, the afterhyperpolarization of action potentials in $\mathrm{IB}^{+}{ }^{+}$was nearly doubled compared to that in $\mathrm{IB}^{-}{ }^{-}$neurons (Figure 1L). Moreover, the action potential in $\mathrm{IB}^{+}$neurons exhibited an apparent afterdepolarization

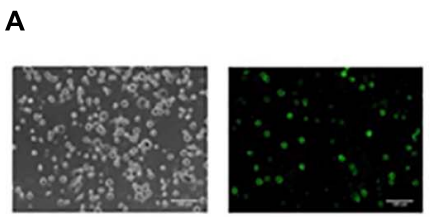

E
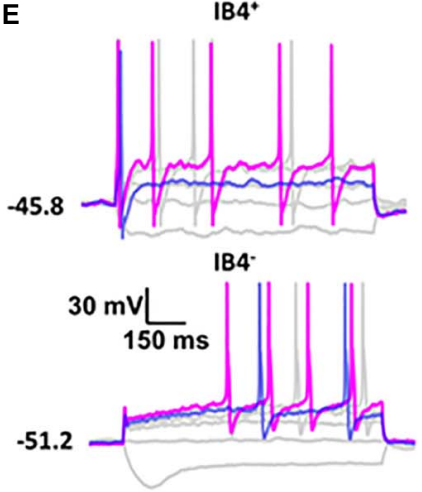

I

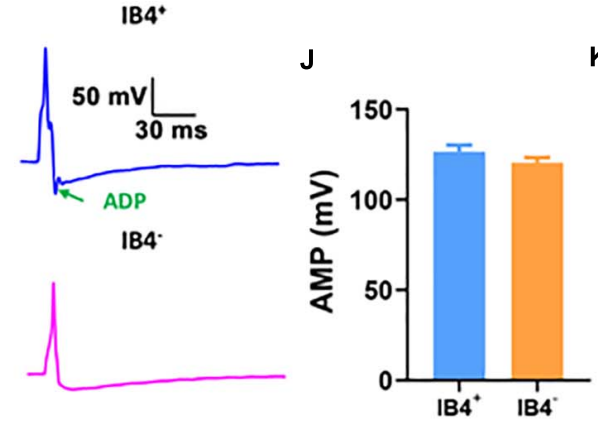

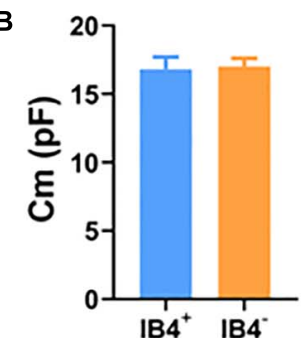

$\mathbf{F}$

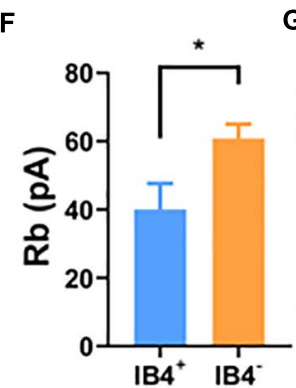

G
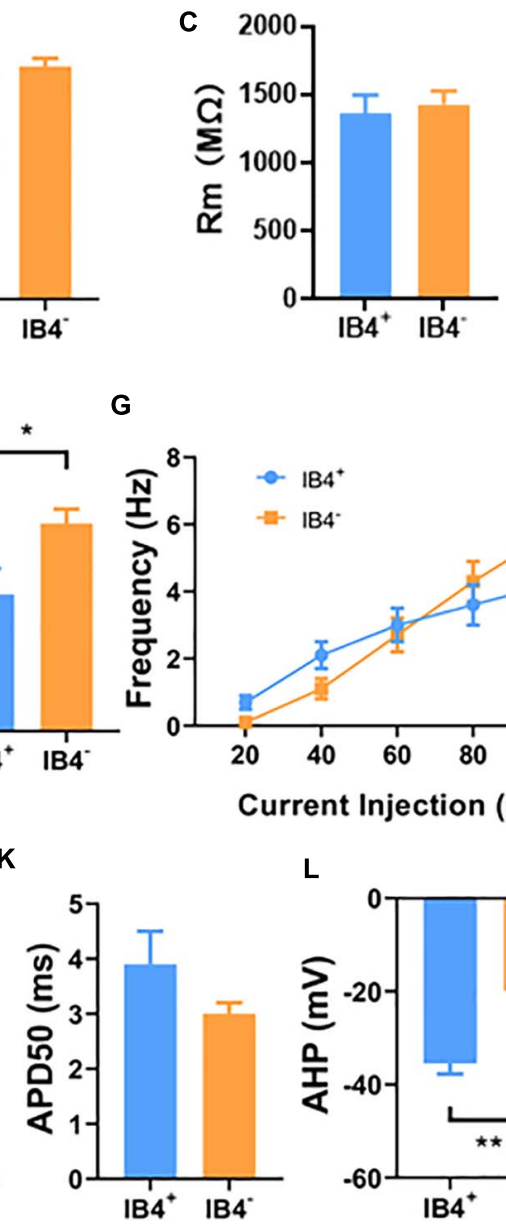

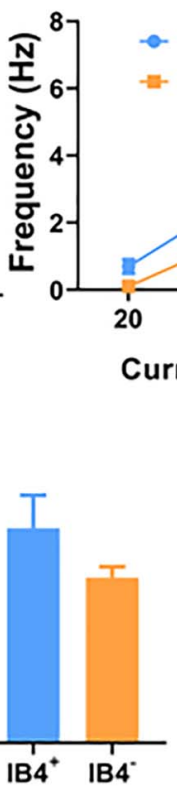

D

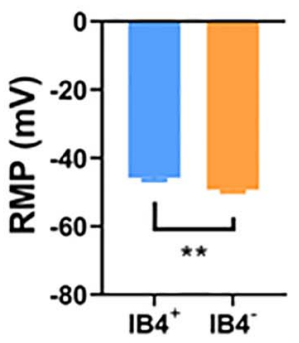

H

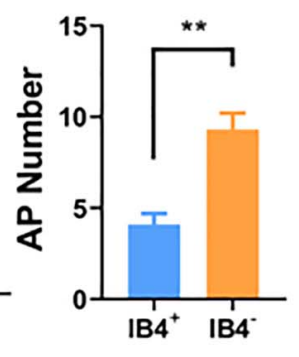

$\mathbf{L}$

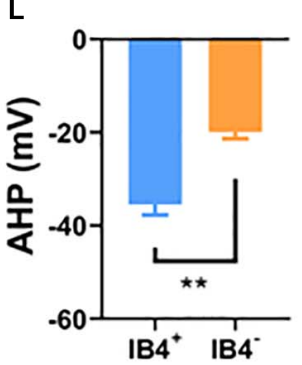

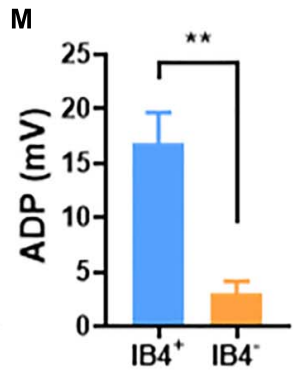

FIGURE 1 | Comparison of passive and active properties of IB4 ${ }^{+}$and IB4 ${ }^{-}$subpopulations of small DRG neurons in mouse. IB4 $4^{+}$and IB4 $4^{-}$subpopulations of small DRG neurons $(<25 \mu \mathrm{m})$, separated by the staining of IB4-FITC (A). Small DRG neurons were recorded in current-clamp mode using a perforated configuration of whole-cell patch clamping. To avoid the confounding effects of IB4 staining on the forming of Giga-seal for patch clamp recording and potentially on the electrophysiological properties of DRG neurons, the DRG neurons were stained immediately after recording. Representative recordings using a long $(1,000 \mathrm{~ms})$ or short (5 ms) step current protocol were shown in (E,I), respectively. Passive properties including membrane capacitance [Cm, (B)], membrane resistance [Rm, (C)], resting membrane potential [RMP,(D)], and active properties including rheobase [Rb, (F)], frequency (G), number at 3x rheobase (H), amplitude [AMP, (J)], half duration [APD50, (K)], afterhyperpolarization [AHP, (L)], and afterdepolarization [ADP, (I,M)] of action potentials were compared between IB4 ${ }^{+}$and IB4 ${ }^{-}$neurons. $n=17-38$ for each groups (see detailed numbers for each group in results). ${ }^{*}, P<0.05$; ${ }^{* *}, P<0.01$; Student's $t$-test. 
potential (ADP) spike, which is often absent in IB4- neurons (Figures 1I,M). Taken together, our results suggest that the electrophysiological properties between mouse $\mathrm{IB}^{+}{ }^{+}$and $\mathrm{IB}_{4}^{-}$ neurons are substantially different.

\section{Oxaliplatin Selectively Depolarized IB4- Neurons, but Not IB4 ${ }^{+}$Neurons}

To assess the effects of oxaliplatin on the electrophysiological properties in the two subgroups of small DRG neurons, $\mathrm{IB}_{4}^{+}$and IB4- DRG neurons were recorded after 15-60 min pre-treatment of oxaliplatin $(50 \mu \mathrm{M})$ in vitro. The cells in control $(n=12$ and 13 for $\mathrm{IB}^{+}{ }^{+}$and $\mathrm{IB}^{-}$neurons, respectively) and oxaliplatin ( $n=8$ and 14 for $\mathrm{IB}^{+}{ }^{+}$and $\mathrm{IB}^{-}$neurons, respectively) groups have similar membrane capacitance for both $\mathrm{IB}_{4}^{+}$and IB4 ${ }^{-}$ groups (Figure $\mathbf{2 A}$ ). In addition, oxaliplatin did not change the membrane resistance (Figure 2B), resting membrane potential (Figure 2C), the rheobase and the firing frequency of action potentials in $\mathrm{IB}^{+}{ }^{+}$neurons significantly (Figures 2D,F,G). In contrast, the membrane resistances of IB4 ${ }^{-}$neurons deceased by $\sim 500 \mathrm{M} \Omega$ after oxaliplatin treatment (Figure 2B). Meanwhile, the resting potentials of $\mathrm{IB}^{-}$neurons were depolarized by $\sim 5.6 \mathrm{mV}$ following oxaliplatin treatment (Figure 2C), which consequently reduced the rheobase current and increased the firing frequency of action potentials trigged by $60-100 \mathrm{pA}$ of injection currents (Figures 2E,F,H). The other parameters of action potentials such as AP threshold, amplitude, half-width, and afterhyperpolarization were not significantly changed by oxaliplatin in both cell subtypes (Table 1). Overall, these results indicate that the excitability of $\mathrm{IB}^{-}$neurons, but not $\mathrm{IB}^{+}$ neurons, were selectively enhanced by acute oxaliplatin treatment in vitro.

\section{Retigabine Prevented Oxaliplatin-Induced Nociceptive Behavior}

To test if the oxaliplatin-induced membrane depolarization of DRG neurons might serve as an initial drive that contributes to the development of oxaliplatin-induced sensory hypersensitivity, we examined the potential preventative effects of retigabine, an opener of potassium channel Kv7 that hyperpolarizes the resting potential of DRG neurons (CorbinLeftwich et al., 2016), on the sensory and motor behaviors in oxaliplatin treated mice.

TABLE 1 | Shape properties of action potentials in the absence and presence of oxaliplatin.

\begin{tabular}{lcccc}
\hline IB4 $^{+}$ & Vt $(\mathbf{m V})$ & AMP $(\mathbf{m V})$ & AHP $(\mathbf{m V})$ & APD50 (ms) \\
\hline Control $(n=12)$ & $-13.8 \pm 4.2$ & $125.9 \pm 4.6$ & $-37.8 \pm 2.8$ & $3.4 \pm 0.2$ \\
Oxaliplatin $(n=8)$ & $-18.1 \pm 3.0$ & $128.7 \pm 6.5$ & $-30.5 \pm 1.4$ & $3.6 \pm 0.6$ \\
IB4- & & & & \\
Control $(n=13)$ & $-17.0 \pm 2.5$ & $118.4 \pm 5.0$ & $-21.9 \pm 2.4$ & $3.4 \pm 0.3$ \\
Oxaliplatin $(n=14)$ & $-12.2 \pm 2.8$ & $112.3 \pm 2.3$ & $-20.6 \pm 2.3$ & $3.5 \pm 0.3$ \\
\hline Vt, voltage threshold; & AMP, & amplitude; & AHP, & afterhyperpolarization; \\
APD50, half duration. & & & &
\end{tabular}

As shown in Figure 3 ( $n=6$ for all groups), oxaliplatin significantly reduced the paw withdrawal threshold to mechanical stimuli (Figure 3A) and increased withdrawal score to cold stimuli (Figure 3B) 3 days after oxaliplatin treatment $(5 \mathrm{mg} / \mathrm{kg}$, ip). In contrast to the mechanical allodynia and cold hyperalgesia, oxaliplatin did not change the paw withdrawal latency to heat stimuli (Figure 3C) or time stayed on rod for the rotarod test (Figure 3D) 4 days following oxaliplatin, in the same groups of animals that had their mechanical and cold tests on Day 3 (Figures 3A,B). Although heat and motor behaviors were tested at Day 4 in these groups of animals in order to compare different types of behaviors in same groups of animals, separate experiments found that heat and motor behaviors were not changed by oxaliplatin at Day 3 as well (data not shown).

To test the potential preventative effects on oxaliplatininduced sensory hypersensitivity, retigabine $(10 \mathrm{mg} / \mathrm{kg}$, ip, daily) was administrated at Day -2, Day -1, Day 0, Day 1, and Day 2. Oxaliplatin was injected at Day 0. As shown in Figure 3, retigabine largely prevented mechanical allodynia (Figure 3A) and significantly attenuated cold hyperalgesia (Figure 3B) caused by oxaliplatin. On the other hand, retigabine did not change heat or motor behaviors significantly (Figures 3C,D).

\section{TRP Channels Mediated the Depolarization Induced by Oxaliplatin}

As the membrane depolarization may be the key electrophysiological effect caused by oxaliplatin that consequently results in the neuronal hyperexcitability (Figure 2), we further studied the possible ion channels that may be involved in the oxaliplatin-induced membrane depolarization. Because the membrane resistance of $\mathrm{IB}^{-}{ }^{-}$neurons was significantly reduced by oxaliplatin along with the membrane depolarization, it is suggested that oxaliplatin increased the permeability of extracellular cations, such as sodium and calcium, at resting state. As TRP channels are a major family of non-selective cation channels expressed in DRG neurons that are directly involved in a variety of chemical and thermal sensing, we tested whether some of the major TRP channel subtypes are involved in the oxaliplatin-induced membrane depolarization. Specific channel antagonists including A-967079 (1 $\mu \mathrm{M})$ (Figure 4A), Capsazepine $(10 \mu \mathrm{M})$ (Figure 4B), and TC-I (10 nM) (Figure 4C), were used to block TRPA1, TRPV1, and TRPM8 channels, respectively.

As shown in Figure 4, pre-treatment of Capsazepine $(n=10$ and 11 for control and oxaliplatin groups, respectively) did not prevent the oxaliplatin-induced changes in membrane resistance (Figure 4D), resting membrane potential (Figure 4E), AP rheobase (Figure 4F). However, in the presence of A967079 ( $n=12$ and 10 for control and oxaliplatin groups, respectively) or TC-I $(n=6$ and 12 for control and oxaliplatin groups, respectively), there were no significant changes in these parameters between control and oxaliplatin groups (Figures $4 \mathrm{D}-\mathrm{F}$ ). These results indicate that TRPA1 and/or TRPM8, but not TRPV1, may contribute to the oxaliplatin-induced membrane depolarization and neuronal sensitization, which might contribute to the initiation of sensory hypersensitivity induced by oxaliplatin. 

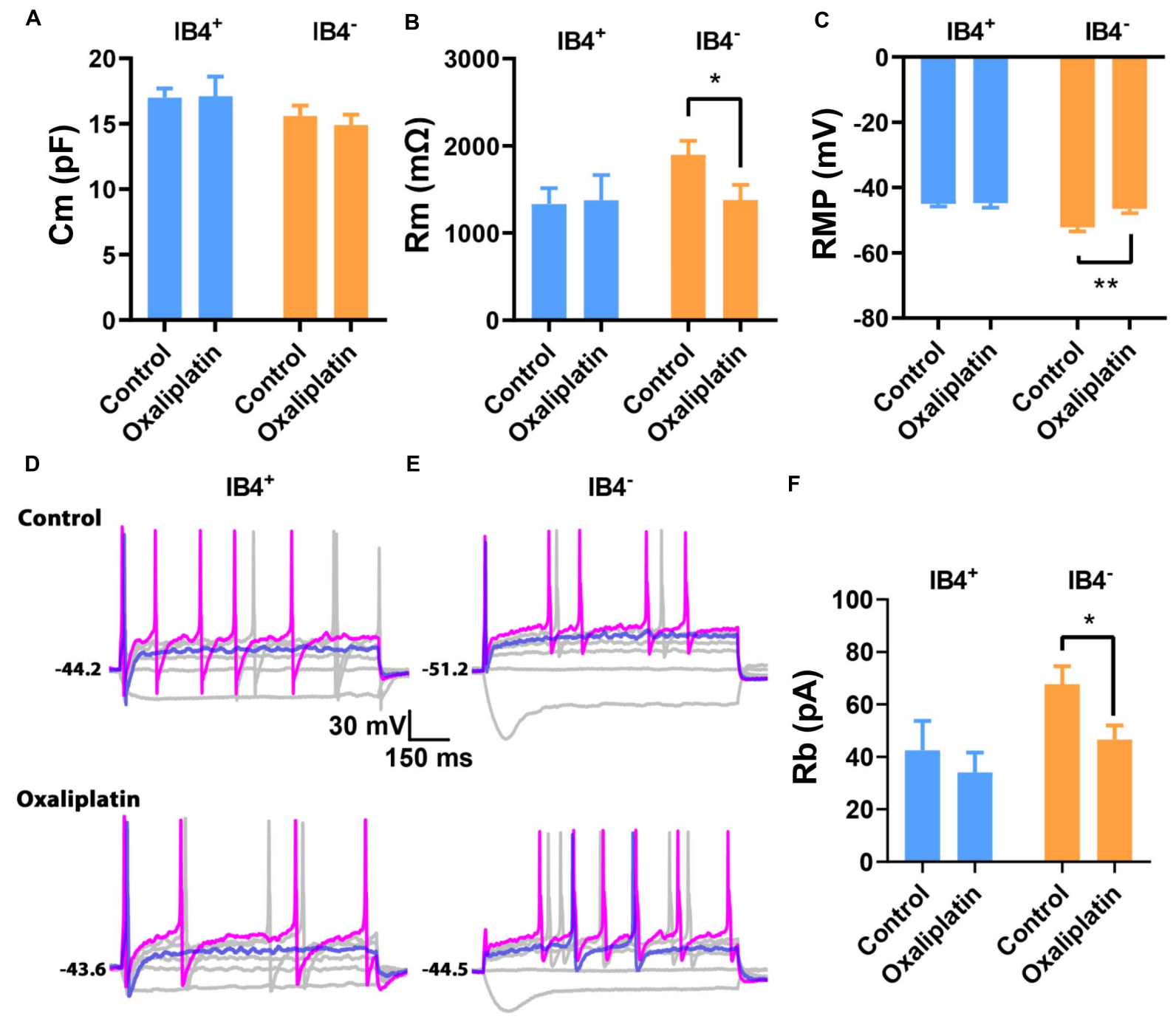

$\mathbf{F}$

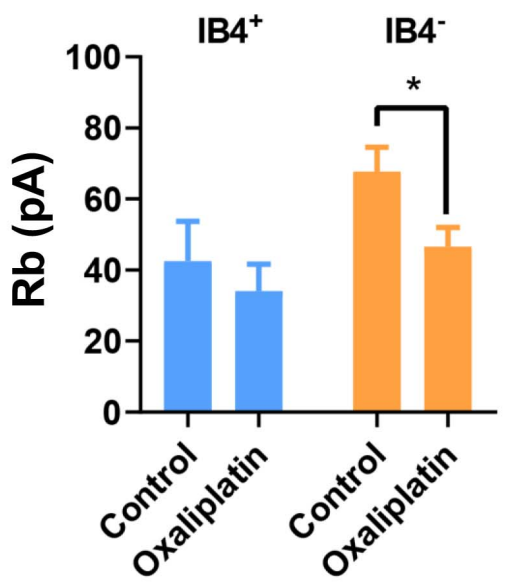

G

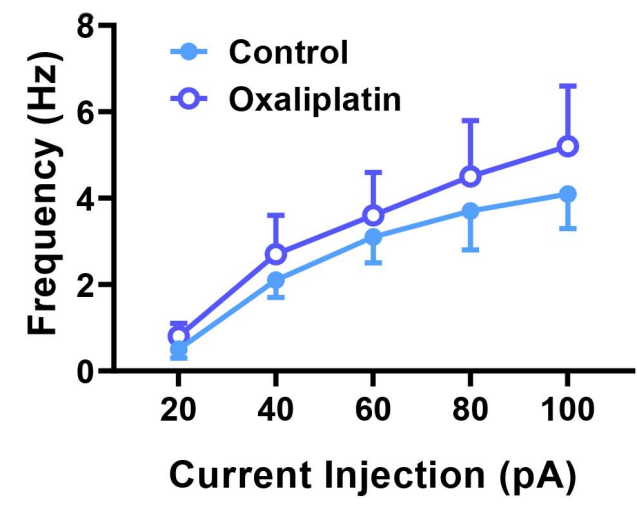

H

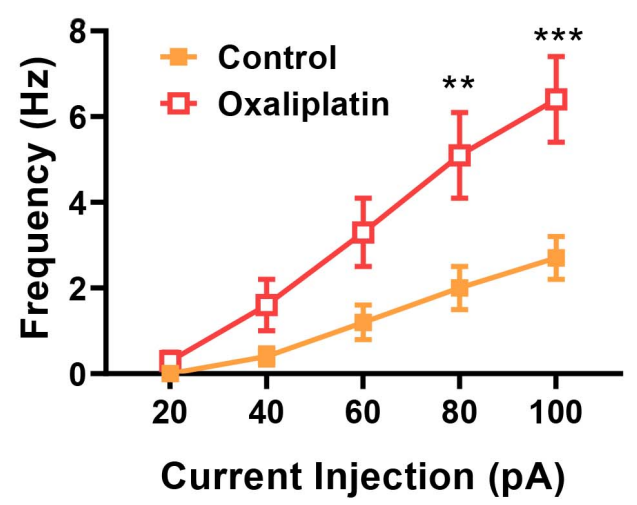

FIGURE 2 | Effects of oxaliplatin on the electrophysiological properties of IB4 ${ }^{+}$and IB4- $4^{-}$subpopulations of small DRG neurons. Oxaliplatin (50 $\mu$ M) was pre-treated in the recording chamber for 15-60 min. The passive and active properties of IB4 ${ }^{+}$and IB4- subpopulations of small DRG neurons were recorded and presented in the same way described in the legend of Figure 1. Oxaliplatin did not change the $\mathrm{Cm}$ of both IB4 ${ }^{+}$and IB4- neurons (A) but decreased the Rm (B) and depolarized the RMP (C) in IB4- neurons; (D,E) Representative recordings from IB4 ${ }^{+}$or IB4- DRG neurons in the absence or presence of oxaliplatin, respectively. (F) The effect

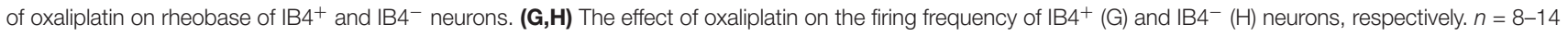
for each group (see detailed numbers for each group in results). ${ }^{\star} P<0.05,{ }^{\star \star} P<0.01,{ }^{\star \star \star} P<0.001$, Student's $t$-test (B,C,F) or Two-way repeated measure ANOVA (H). 
A

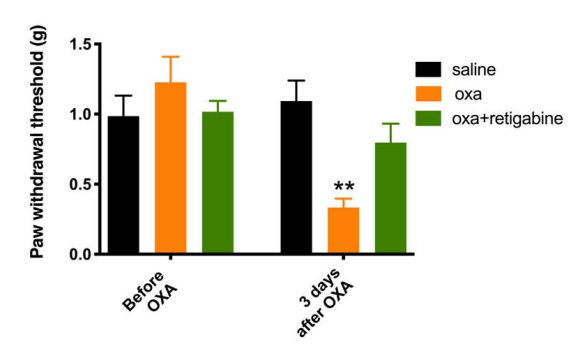

C

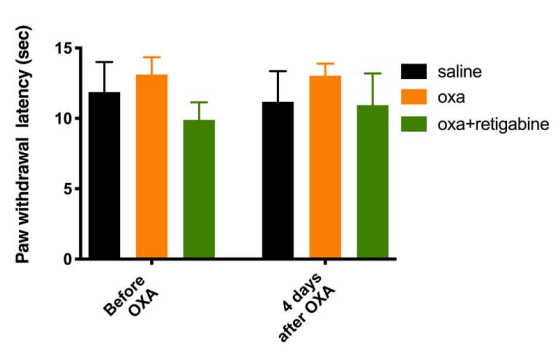

B

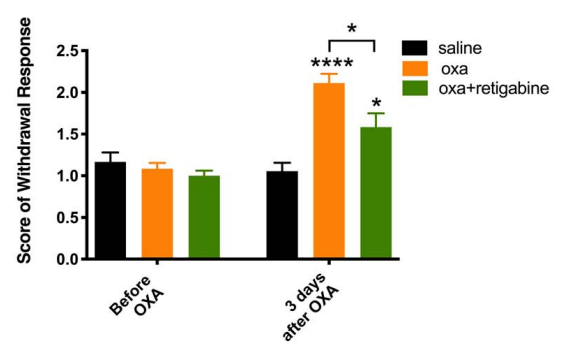

D

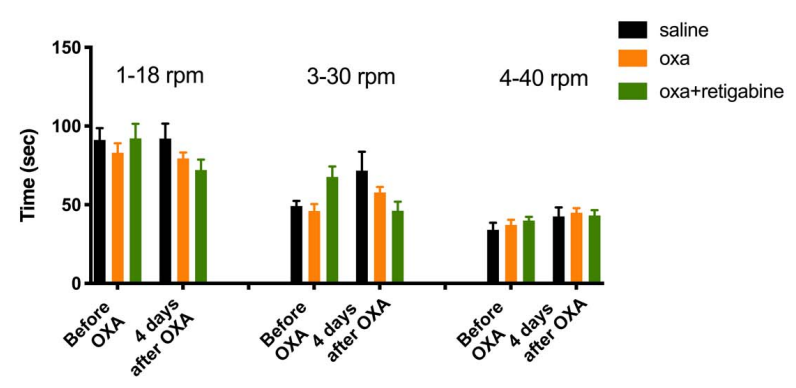

FIGURE 3 | Effects of retigabine on the sensory and motor behaviors following oxaliplatin treatment. Oxaliplatin (oxa, 5 mg/kg, ip) was administrated at Day 0. Retigabine (10 mg/kg, ip, daily) was treated at Day -2, Day -1, Day 0, Day 1, and Day 2. The same groups of animals were tested for mechanical and cold behaviors at Day 3, and were tested for heat and motor behaviors at Day 4. (A) Paw withdrawal threshold to Von-Frey filament; (B) Score of withdrawal response to acetone; (C) Paw withdrawal latency to radiant heat; and (D) Time stayed on rod at different speeds in rotarod tests $\left(n=6\right.$ for all groups). ${ }^{*}, P<0.05$; ${ }^{*}, P<0.01$;

$* \star \star *, P<0.0001$; two-way repeated measure ANOVA.

\section{TC-I, but Not A-967079, Prevented Oxaliplatin-Induced Nociceptive Behavior}

To test if TRPM8 and TRPA1 might be involved in the initial pain-driving process of the oxaliplatin-induced sensory hypersensitivity, we examined the potential preventative effects of TC-I and A-967079 on the sensory and motor behaviors in oxaliplatin treated mice.

As shown in Figure 5 ( $n=6$ for all groups), daily treatment of $10 \mathrm{mg} / \mathrm{kg}$ TC-I (ip, administrated at Day -2, Day -1, Day 0 , Day 1, and Day 2) largely prevented mechanical and cold hypersensitivity induced by oxaliplatin administrated at Day 0 (Figures 5A,B) without causing any changes in the heat and motor behaviors tested (Figures 5C,D). In contrast, the treatment of $100 \mathrm{mg} / \mathrm{kg} \mathrm{A-967079}$ (po) with the same schedule of TC-I did not change any behaviors following oxaliplatin administration (Figure 6) ( $n=6$ for all groups). These results suggest that TRPM8 may play a critical role in the initiation of sensory hypersensitivity induced by oxaliplatin.

\section{DISCUSSION}

In the present study, we found that there were previously undiscovered, substantial differences in membrane excitability between $\mathrm{IB}^{+}$and $\mathrm{IB}^{-}$subpopulations of small-sized DRG neurons in mice. These differences included depolarized resting membrane potential, lower rheobase of the action potential, overwhelmingly larger afterdepolarization, and nearly doubled afterhyperpolarization in $\mathrm{IB}^{+}$neurons compared to $\mathrm{IB}^{-}$ neurons (Figure 1). Differential properties of membrane excitability have been reported previously between $\mathrm{IB}^{+}$and IB4 ${ }^{-}$subpopulations of small DRG neurons of rats and mice (Stucky and Lewin, 1999; Wu and Pan, 2004; Choi et al., 2007; Zhang et al., 2010). These differences, in IB4 ${ }^{+}$compared to IB4 ${ }^{-}$ subpopulation of neurons, included a longer duration of the action potential in both rat and mouse neurons, a hyperpolarized resting membrane potential and/or higher rheobases of action potentials in rat neurons, and a smaller afterhyperpolarization in cutaneous rat neurons. Interestingly, none of the major findings in the present study have been reported previously. A possible reason for the different findings between previous and current studies could be due to the different recording configuration of whole-cell patch clamping. Typical whole-cell recordings were used in the previous studies while a perforated wholecell recording was used in the present study. As the perforation introduced by amphotericin B does not allow the exchange of ions and molecules larger than monovalent ions between patch pipettes and cytosols of cells, divalent ions such as intracellular $\mathrm{Ca}^{2+}$, and other cytosolic molecules might contribute to these differences. For example, a predominant expression of large conductance, calcium activated potassium currents $\left(\mathrm{BK}_{\mathrm{Ca}}\right)$ was discovered in $\mathrm{IB}^{+}{ }^{+}$cutaneous DRG neurons which would result 
A

A

A-967097

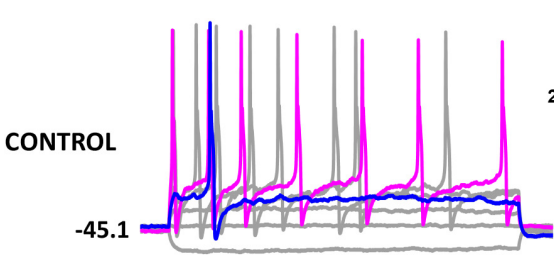

B

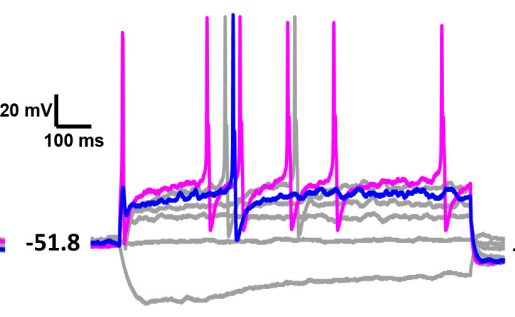

C TC-I

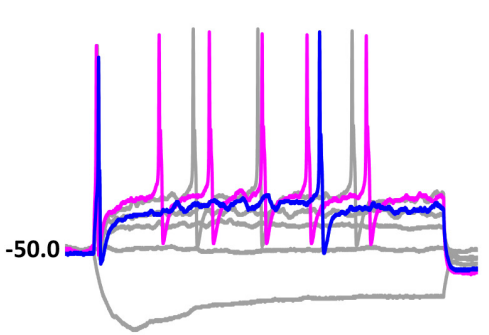

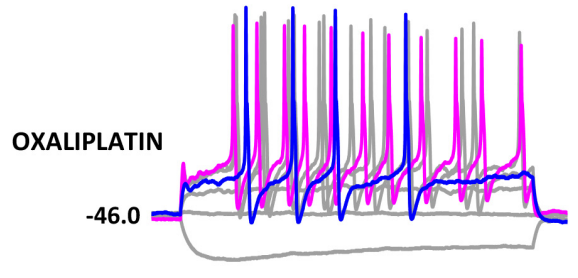

D

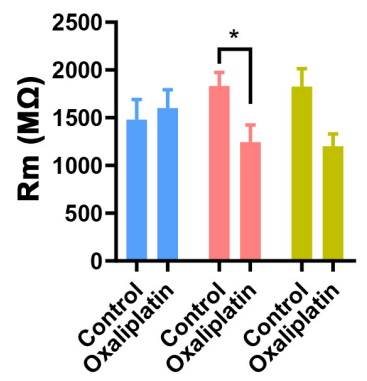

E

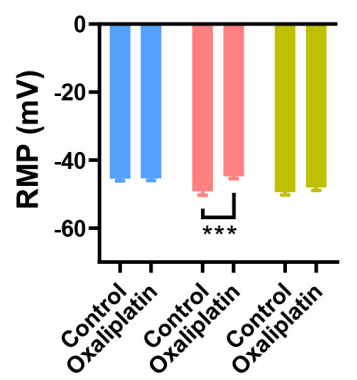

$\mathbf{F}$

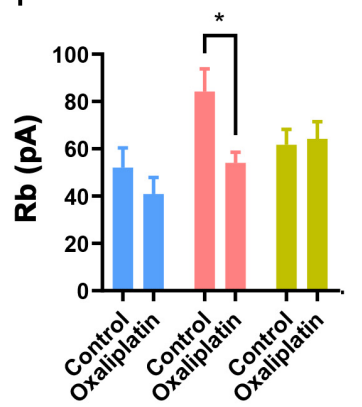

G

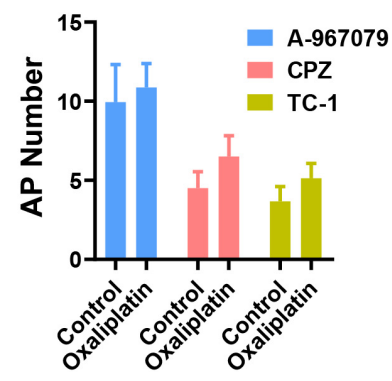

FIGURE 4 | Effects of selective TRP channel antagonists on the electrophysiological properties of IB4 ${ }^{+}$and IB4- $^{-}$subpopulations of small DRG neurons. Selective antagonists for TRPA1 (1 $\mu \mathrm{M}$ A-967097), TRPV1 (10 $\mu \mathrm{M}$ Capsazepine, CPZ), or TRPM8 (10 nM TC-I) were pre-treated in the recording chamber for 15 min before the addition of $50 \mu \mathrm{M}$ oxaliplatin. The passive and active properties of IB4 ${ }^{+}$and IB4- ${ }^{-}$subpopulations of small DRG neurons were recorded and presented in the same way described in the legend of Figure 1. (A-C) Representative recordings in the absence or presence of oxaliplatin, and in the presence of A-967097, Capsazepine, and TC-I, respectively. Effect of oxaliplatin on Rm (D), RMP (E) and Rb (F) in each group of TRP antagonists. (G) Number of action potentials evoked by 100 pA current injection in each group of cells. $n=6-12$ for each group (see detailed numbers for each group in results). ${ }^{\star} P<0.05$, ${ }^{\star \star \star} P<0.001$, Student's $t$-test.

in a larger afterhyperpolarization in these neurons (Zhang et al., 2010). However, a smaller afterhyperpolarization was actually observed in these neurons using typical whole-cell recording (Zhang et al., 2010). On the other hand, using perforated whole-cell recording, we found that there were a nearly doubled afterhyperpolarization expressed in the $\mathrm{IB}^{+}$ DRG neurons (Figures 1I,L). Therefore, the revealing of the larger afterhyperpolarization in $\mathrm{IB}^{+}$neurons is likely due to the perforated whole-cell recording used in the current study that allowed the endogenous $\mathrm{Ca}^{2+}$ dynamics unbuffered by the $\mathrm{Ca}^{2+}$ chelators included in the patch pipette. In addition to the different configurations of whole-cell recordings, other factors such as species of rodents, mouse strains, culture protocols could also contribute to the differences among previous and current studies.

Among the major differential membrane properties between $\mathrm{IB}_{4}^{+}$and $\mathrm{IB}^{-}{ }^{-}$neurons found in the current study, the afterdepolarization recorded in the small DRG neurons has not been reported (Figures $\mathbf{1 I}, \mathbf{M}$ ). Previously a different type of afterdepolarization was reported in the subpopulations of medium, or small-to-medium DRG neurons that are enriched with T-type calcium channels $\left(\mathrm{Ca}_{\mathrm{T}}\right)$ in rats (White et al., 1989; Nelson et al., 2005). However, the afterdepolarization recorded from $\mathrm{Ca}_{\mathrm{T}}$-enriched neurons did not appear at the resting membrane potential and a membrane hyperpolarization was needed to induce this type of afterdepolarization. On the other hand, afterdepolarization recorded in the small mouse neurons of the present study was induced at normal resting membrane potential. Moreover, the afterdepolarization was much shorter in the present study compared to that recorded in the previous studies. It has been reported that a variety of ion currents can contribute to afterdepolarization in neurons. These ionic mechanisms include low threshold sodium currents, nonselective cationic currents, KCNQ/M channels, calcium-activated chloride currents, and sodium-calcium exchanger (Higashi et al., 1993; Linden et al., 1994; Azouz et al., 1996; Haj-Dahmane and Andrade, 1998; Yue and Yaari, 2004; Ghitani et al., 2016). As it would beyond the scope of the current study, future studies are needed to study the ionic mechanism of the novel afterdepolarization recorded in the present study. 
A

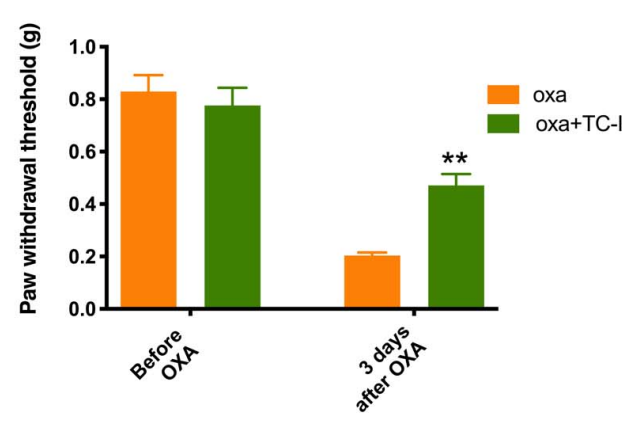

C

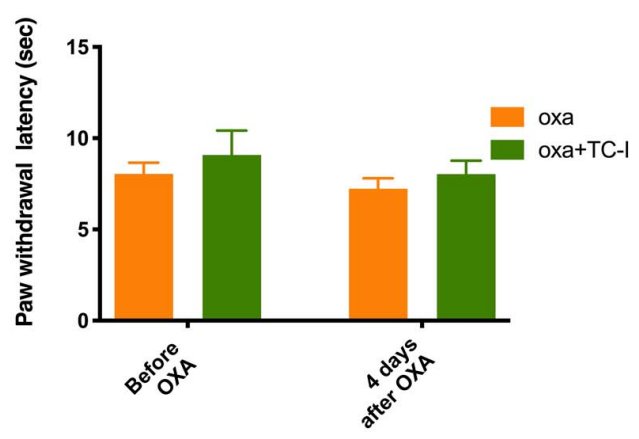

B

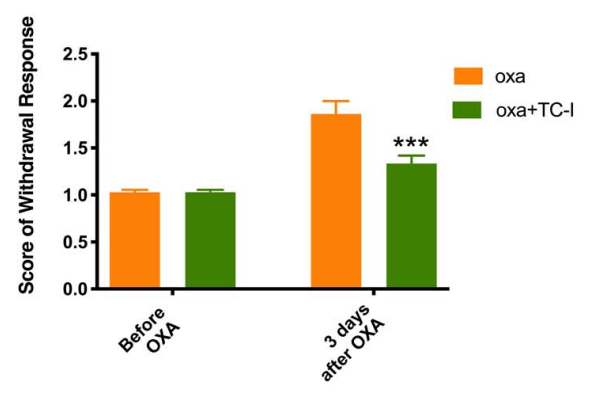

D

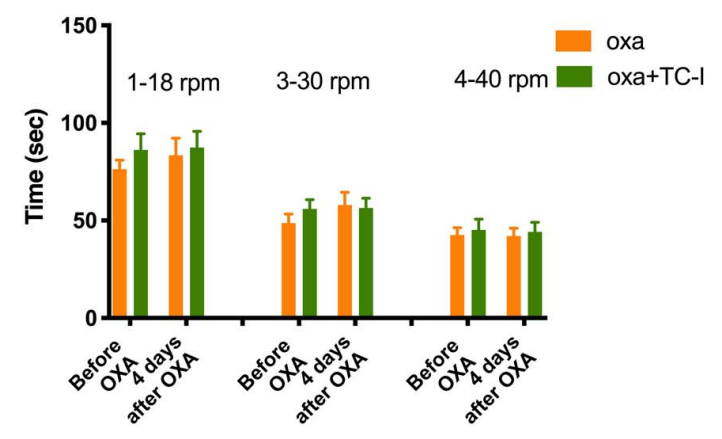

FIGURE 5 | Effects of TC-I on the sensory and motor behaviors following oxaliplatin treatment. The administration of oxaliplatin, the schedule for TC-I (10 mg/kg, ip) treatment (same to the Retigabine treatment), the testing schedule for different types of behaviors were described in the Legend of Figure 3. (A) Paw withdrawal threshold to Von-Frey filament; (B) Score of withdrawal response to acetone; (C) Paw withdrawal latency to radiant heat; and (D) Time stayed on rod at different speeds in rotarod tests ( $n=6$ for all groups). ${ }^{\star \star}, P<0.01$; ${ }^{\star \star \star \star}, P<0.0001$; two-way repeated measure ANOVA.

One major finding of this study is that in vitro oxaliplatin selectively depolarized and increased the membrane excitability of the $\mathrm{IB}^{-}{ }^{-}$, but not $\mathrm{IB}^{+}{ }^{+}$small DRG neurons. Previously, membrane depolarization and reduction in rheobases induced by in vitro oxaliplatin have been reported (Cerles et al., 2019; Zhang et al., 2021). However, a selective effect of in vitro oxaliplatin on the membrane excitability of IB $4^{-}$over IB $4^{+}$neurons has not been reported. As the two major subpopulations of DRG neurons, $\mathrm{IB}^{-}{ }^{-}$and $\mathrm{IB}_{4}{ }^{+}$neurons are largely different in their expression of neuronal peptides, peripheral innervation and central projection in spinal cord, modulation by growth factors, expression of ion channels and membrane receptors, and physiological function (Verge et al., 1989; Silverman and Kruger, 1990; Molliver et al., 1997; Zylka et al., 2005; Jankowski et al., 2009; Chiu et al., 2014). Therefore, a selective excitatory effect of oxaliplatin on the IB4 ${ }^{-}$subpopulation of small DRG neurons would selectively activate peptidergic innervation at periphery and therefore their central projections at Lamina I and outer Lamina II in the spinal cord.

In the present study, we found that a membranehyperpolarizing drug retigabine largely prevented the oxaliplatin-induced mechanical and cold hyperalgesia (Du et al., 2014). A previous study also showed that retigabine prevented oxaliplatin-induced orofacial cold hyperalgesia that is likely involved with TRP channels also (Abd-Elsayed et al., 2015; Luo et al., 2021). As retigabine opens potassium channels at resting membrane potential leading to membrane hyperpolarization, these results suggest that targeting oxaliplatininduced membrane depolarization might be a useful strategy to prevent oxaliplatin-induced sensory hyperalgesia.

Another finding of the in vitro study is that blockade of TRPA1 and TRPM8, but not TRPV1 prevented the oxaliplatin-induced membrane depolarization and reduction in the rheobase of action potentials (Figure 4). These results suggest that TRPA1 and TRPM8, but not TRPV1 contribute to oxaliplatin-induced membrane depolarization in $\mathrm{IB}^{-}$ subpopulation of small DRG neurons in mice. Previous it has been reported that in vitro oxaliplatin potentiates the increasing effects of agonists of TRPA1 and TRPV1, but not TRPM8 on intracellular $\mathrm{Ca}^{2+}$ in DRG neurons (Anand et al., 2010; Nassini et al., 2011; Zhao et al., 2012). The consistent (for TRPA1) and different (for TRPV1 and TRPM8) effects of TRP channel agonists on the membrane excitability compared to intracellular $\mathrm{Ca}^{2+}$ suggest that oxaliplatin might induce membrane hyperexcitability and increased intracellular $\mathrm{Ca}^{2+}$ through partially overlapping mechanisms in RG neurons.

Upregulation of multiple TRP channel isoforms (TRPA1, TRPV1, and TRPM8) have been reported following oxaliplatin treatment (Chukyo et al., 2018). The upregulation of these 
A

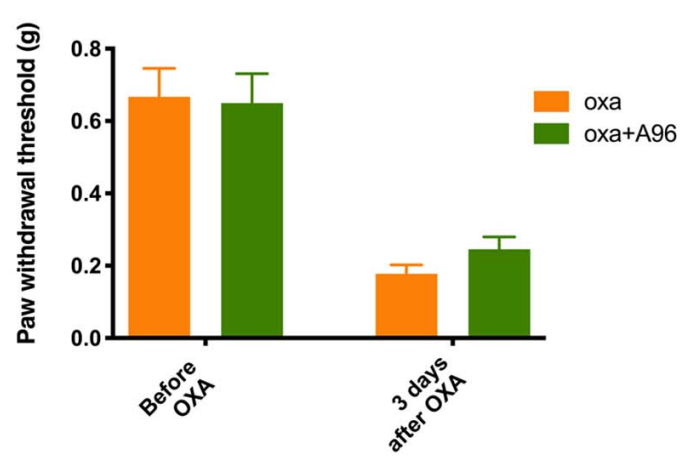

C

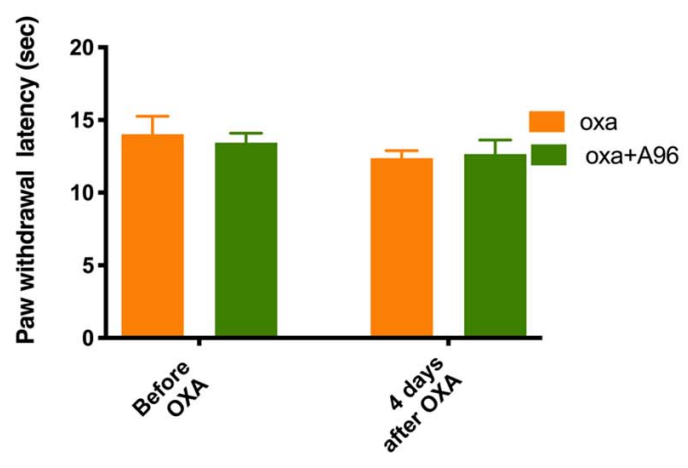

B

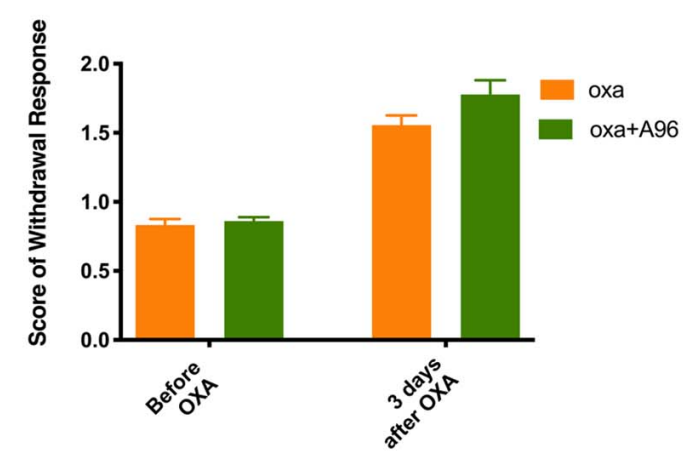

D

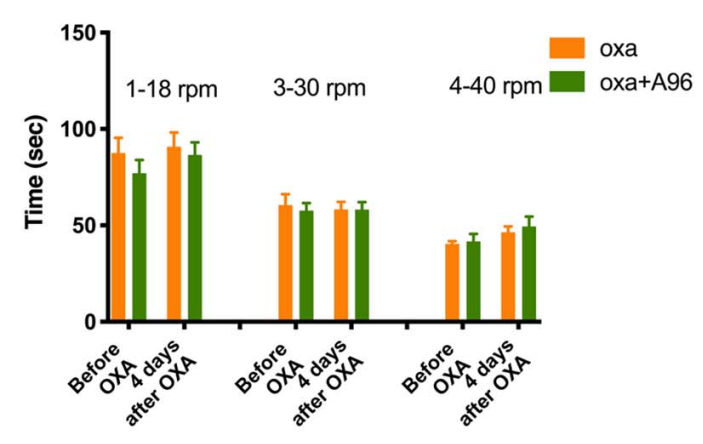

FIGURE 6 | Effects of A-967079 on the sensory and motor behaviors following oxaliplatin treatment. The administration of oxaliplatin, the schedule for A-967079 (A-96, $100 \mathrm{mg} / \mathrm{kg}, \mathrm{po}$ ) treatment (same to the Retigabine and TC-I treatment), the testing schedule for different types of behaviors were described in the Legend of Figure 3. (A) Paw withdrawal threshold to Von-Frey filament; (B) Score of withdrawal response to acetone; (C) Paw withdrawal latency to radiant heat; and (D) Time stayed on rod at different speeds in rotarod tests ( $n=6$ for all groups).

TRP channels following oxaliplatin treatment may or may not contribute to the oxaliplatin-induced mechanical and/or cold hyperalgesia (Gauchan et al., 2009; Mizuno et al., 2014; Chen et al., 2015; Yamamoto et al., 2015). Different from previous studies, the current study is focused on the potential role of TRP channels and membrane depolarization as the initial ionic/membrane drives (such as within an hour) that contribute to the development of oxaliplatin-induced neuropathic pain. Our results suggest that TRPM8 and TRPM8mediated membrane depolarization, but not TRPA1 or TRPV1, serve as the initial ionic/membrane drives that contribute to the development of oxaliplatin-induced neuropathic pain. On the other hand, although the current study might provide some new suggestion on the role of TRPM8 as the initial ionic drive for the development of oxaliplatin-induced acute pain, we fully recognize that the potential TRPM8 involvement in Oxaliplatininduced neurotoxicity has been reported by other researchers previously (Gauchan et al., 2009; Ta et al., 2010; Descoeur et al., 2011; Kono et al., 2012; Mizuno et al., 2014; Hsieh et al., 2017; Chukyo et al., 2018).

Although most studies of TRPM8 and allodynia have been focused on the cold allodynia, the involvement of TRPM8 in mechanical allodynia has been reported in multiple inflammatory and neuropathic pain conditions (Gao et al., 2013; Caceres et al., 2017; De Caro et al., 2018). The current manuscript showed that TRPM8 also participant in the oxaliplatin induced mechanical allodynia in addition to the cold allodynia. However, a previous study found that knockout of TRPM8 did not prevent the development of mechanical hyperalgesia induced by oxaliplatin (Descoeur et al., 2011). This discrepancy might be due to the different mechanical force used for the vonFrey tests. The previous study used $1.4 \mathrm{~g}$ as the stimulating force while the current study found that the mechanical threshold changed from about 0.2 to $0.45 \mathrm{~g}$ in the absence and presence of TC-I pre-treatment. Moreover, it has been reported that a selective reduction of TRPM8, but not TRPV1 is associated with the transcutaneous ultrasound nerve stimulation-induced inhibition of mechanical and cold allodynia following oxaliplatin treatment (Hsieh et al., 2017). Therefore, it might be suggested that TRPM8 is more involved in the mechanical allodynia compared to mechanical hyperalgesia induced by oxaliplatin.

One inconsistence in results in the present study is that the TRPA1 antagonist prevented oxaliplatin-induced membrane depolarization in DRG neurons in vitro but did not prevent the oxaliplatin-induced neuropathic pain behaviors in vivo. 
One possibility for this discrepancy could be due to the dissociated cell culture of DRG neurons. It has been suggested that dissociation of DRG neurons might induce hyperexcitability that is caused by increased PKA and PKG signaling (Zheng et al., 2007). As PKA can sensitize TRPA1 channels, the TRPA1 in vitro might become more sensitive to oxaliplatin through ROS or other mechanisms compared to in vivo (Meents et al., 2017; Zheng et al., 2019). Another possibility might be that TRPA1 and TRPM8 are functionally coupled in the dissociated DRG neurons but not in vivo, as association of different types of TRP channels has been reported in DRG neurons (Patil et al., 2020). Nevertheless, other possibilities can not be excluded and future studies are needed to address this difference.

The present study suggests that oxaliplatin depolarizes IB4 ${ }^{-}$ DRG neurons through activation of TRPM8 channels to drive the development of neuropathic pain in mice. However, a previous study suggested that oxaliplatin acts on $\mathrm{IB}^{+}{ }^{+}$nociceptors to induce peripheral sensory neuropathy (Joseph et al., 2008). Specifically, the previous study found that intrathecal IB4-saporin prevented mechanical hyperalgesia induced by oxaliplatin while the present study found that the antagonist of TRPM8 (not expressed in $\mathrm{IB}^{+}{ }^{+}$neurons) prevented both mechanical and cold hyperalgesia induced by oxaliplatin. Taken these results together, it is possible that oxaliplatin-induced mechanical hyperalgesia might be dependent on the activation of both $\mathrm{IB}_{4}^{+}$and IB4subpopulations of DRG neurons. On the other hand, in addition to the different blocking approach, there are multiple other conditions that might contribute to the different suggestions made from the previous and current studies. These differences include species of experimental animals (rat vs. mouse), route of administration of oxaliplatin (iv vs. ip), types of relevant behavioral tests (mechanical vs. mechanical and cold), and time points of relevant behavioral tests (6-10 vs. 3 days following oxaliplatin treatment).

\section{REFERENCES}

Abd-Elsayed, A. A., Ikeda, R., Jia, Z., Ling, J., Zuo, X., Li, M., et al. (2015). KCNQ channels in nociceptive cold-sensing trigeminal ganglion neurons as therapeutic targets for treating orofacial cold hyperalgesia. Mol. Pain 11:45. doi: 10.1186/s12990-015-0048-8

Anand, U., Otto, W. R., and Anand, P. (2010). Sensitization of capsaicin and icilin responses in oxaliplatin treated adult rat DRG neurons. Mol. Pain 6:82. doi: 10.1186/1744-8069-6-82

Argyriou, A. A., Zolota, V., Kyriakopoulou, O., and Kalofonos, H. P. (2010). Toxic peripheral neuropathy associated with commonly used chemotherapeutic agents. J. BUON. 15, 435-446.

Azouz, R., Jensen, M. S., and Yaari, Y. (1996). Ionic basis of spike after-depolarization and burst generation in adult rat hippocampal CA1 pyramidal cells. J. Physiol. 492(Pt 1), 211-223. doi: 10.1113/jphysiol.1996. sp021302

Beijers, A. J., Mols, F., and Vreugdenhil, G. A. (2014). systematic review on chronic oxaliplatin-induced peripheral neuropathy and the relation with oxaliplatin administration. Support Care Cancer 22, 1999-2007. doi: 10.1007/s00520-0142242-z

Boyette-Davis, J. A., Walters, E. T., and Dougherty, P. M. (2015). Mechanisms involved in the development of chemotherapy-induced neuropathy. Pain Manag. 5, 285-296. doi: 10.2217/pmt.15.19

Briani, C., Argyriou, A. A., Izquierdo, C., Velasco, R., Campagnolo, M., Alberti, P., et al. (2014). Long-term course of oxaliplatin-induced polyneuropathy: a

\section{DATA AVAILABILITY STATEMENT}

The original contributions presented in the study are included in the article/supplementary material, further inquiries can be directed to the corresponding author/s.

\section{ETHICS STATEMENT}

The animal study was reviewed and approved by the Institutional Animal Care and Use Committees of the Indiana University School of Medicine.

\section{AUTHOR CONTRIBUTIONS}

BW, XF, Y-HJ, and Z-YT conceived the idea. BW, XS, and $\mathrm{Z}$-YT designed the experiments, analyzed the data, and wrote the manuscript. BW, XS, WZ, and Y-HZ conducted the experiments. All authors were involved in the discussion of the project.

\section{FUNDING}

Z-YT was supported by a DoD PRMRP DA grant (W81XWH-201-0138), and an NIH R01 grant (\#NS102415). This publication was partially supported by an award from the Indiana University School of Medicine.

\section{ACKNOWLEDGMENTS}

The authors thank Drs. Naikui Liu and Heqiao Dai from Dr. Xiao-Ming Xu's lab for the assistance of rotarod test.

prospective 2-year follow-up study. J. Peripher. Nerv. Syst. 19, 299-306. doi: $10.1111 /$ jns. 12097

Caceres, A. I., Liu, B., Jabba, S. V., Achanta, S., Morris, J. B., and Jordt, S. E. (2017). Transient Receptor Potential Cation Channel Subfamily M Member 8 channels mediate the anti-inflammatory effects of eucalyptol. Br. J. Pharmacol. 174, 867-879. doi: 10.1111/bph.13760

Cerles, O., Goncalves, T. C., Chouzenoux, S., Benoit, E., Schmitt, A., Bennett Saidu, N. E., et al. (2019). Preventive action of benztropine on platinum-induced peripheral neuropathies and tumor growth. Acta Neuropathol. Commun. 7:9. doi: 10.1186/s40478-019-0657-y

Chen, K., Zhang, Z. F., Liao, M. F., Yao, W. L., Wang, J., and Wang, X. R. (2015). Blocking PAR2 attenuates oxaliplatin-induced neuropathic pain via TRPV1 and releases of substance P and CGRP in superficial dorsal horn of spinal cord. J. Neurol. Sci. 352, 62-67. doi: 10.1016/j.jns.2015.03.029

Chiu, I. M., Barrett, L. B., Williams, E. K., Strochlic, D. E., Lee, S., Weyer, A. D., et al. (2014). Transcriptional profiling at whole population and single cell levels reveals somatosensory neuron molecular diversity. Elife 3:4660. doi: 10.7554/ eLife. 04660

Choi, J. S., Dib-Hajj, S. D., and Waxman, S. G. (2007). Differential slow inactivation and use-dependent inhibition of Nav1.8 channels contribute to distinct firing properties in IB4+ and IB4- DRG neurons. J. Neurophysiol. 97, 1258-1265. doi: 10.1152/jn.01033.2006

Chukyo, A., Chiba, T., Kambe, T., Yamamoto, K., Kawakami, K., Taguchi, K., et al. (2018). Oxaliplatin-induced changes in expression of transient receptor potential channels in the dorsal root ganglion as a neuropathic mechanism 
for cold hypersensitivity. Neuropeptides 67, 95-101. doi: 10.1016/j.npep.2017. 12.002

Corbin-Leftwich, A., Mossadeq, S. M., Ha, J., Ruchala, I., Le, A. H., and VillalbaGalea, C. A. (2016). Retigabine holds KV7 channels open and stabilizes the resting potential. J. Gen. Physiol. 147, 229-241. doi: 10.1085/jgp.201511517

De Caro, C., Russo, R., Avagliano, C., Cristiano, C., Calignano, A., Aramini, A., et al. (2018). Antinociceptive effect of two novel transient receptor potential melastatin 8 antagonists in acute and chronic pain models in rat. $\mathrm{Br}$. J. Pharmacol. 175, 1691-1706. doi: 10.1111/bph.14177

de Gramont, A., Figer, A., Seymour, M., Homerin, M., Hmissi, A., Cassidy, J., et al. (2000). Leucovorin and fluorouracil with or without oxaliplatin as firstline treatment in advanced colorectal cancer. J. Clin. Oncol. 18, 2938-2947. doi: 10.1200/JCO.2000.18.16.2938

Descoeur, J., Pereira, V., Pizzoccaro, A., Francois, A., Ling, B., Maffre, V., et al. (2011). Oxaliplatin-induced cold hypersensitivity is due to remodelling of ion channel expression in nociceptors. EMBO Mol. Med. 3, 266-278. doi: 10.1002/ emmm.201100134

Dirajlal, S., Pauers, L. E., and Stucky, C. L. (2003). Differential response properties of IB(4)-positive and -negative unmyelinated sensory neurons to protons and capsaicin. J. Neurophysiol. 89, 513-524. doi: 10.1152/jn.00371.2002

Du, X., Hao, H., Gigout, S., Huang, D., Yang, Y., Li, L., et al. (2014). Control of somatic membrane potential in nociceptive neurons and its implications for peripheral nociceptive transmission. Pain 155, 2306-2322. doi: 10.1016/j.pain. 2014.08.025

Gao, T., Hao, J., Wiesenfeld-Hallin, Z., and Xu, X. J. (2013). Activation of TRPM8 cold receptor triggers allodynia-like behavior in spinally injured rats. Scand. J. Pain 4, 33-37. doi: 10.1016/j.sjpain.2012.09.007

Gauchan, P., Andoh, T., Kato, A., and Kuraishi, Y. (2009). Involvement of increased expression of transient receptor potential melastatin 8 in oxaliplatin-induced cold allodynia in mice. Neurosci. Lett. 458, 93-95. doi: 10.1016/j.neulet.2009.04. 029

Gebremedhn, E. G., Shortland, P. J., and Mahns, D. A. (2018). The incidence of acute oxaliplatin-induced neuropathy and its impact on treatment in the first cycle: a systematic review. BMC Cancer 18:410. doi: 10.1186/s12885-0184185-0

Ghitani, N., Bayguinov, P. O., Basso, M. A., and Jackson, M. B. A. (2016). sodium afterdepolarization in rat superior colliculus neurons and its contribution to population activity. J. Neurophysiol. 116, 191-200. doi: 10.1152/jn.01138.2015

Haj-Dahmane, S., and Andrade, R. (1998). Ionic mechanism of the slow afterdepolarization induced by muscarinic receptor activation in rat prefrontal cortex. J. Neurophysiol. 80, 1197-1210. doi: 10.1152/jn.1998.80.3.1197

Higashi, H., Tanaka, E., Inokuchi, H., and Nishi, S. (1993). Ionic mechanisms underlying the depolarizing and hyperpolarizing afterpotentials of single spike in guinea-pig cingulate cortical neurons. Neuroscience 55, 129-138. doi: 10. 1016/0306-4522(93)90460-w

Hsieh, Y. L., Chen, H. Y., Yang, C. H., and Yang, C. C. (2017). Analgesic Effects of Transcutaneous Ultrasound Nerve Stimulation in a Rat Model of OxaliplatinInduced Mechanical Hyperalgesia and Cold Allodynia. Ultrasound Med. Biol. 43, 1466-1475. doi: 10.1016/j.ultrasmedbio.2017.03.002

Jankowski, M. P., Lawson, J. J., McIlwrath, S. L., Rau, K. K., Anderson, C. E., Albers, K. M., et al. (2009). Sensitization of cutaneous nociceptors after nerve transection and regeneration: possible role of target-derived neurotrophic factor signaling. J. Neurosci. 29, 1636-1647. doi: 10.1523/JNEUROSCI.3474-08. 2009

Joseph, E. K., Chen, X., Bogen, O., and Levine, J. D. (2008). Oxaliplatin acts on IB4-positive nociceptors to induce an oxidative stress-dependent acute painful peripheral neuropathy. J. Pain 9, 463-472. doi: 10.1016/j.jpain.2008. 01.335

Kerckhove, N., Collin, A., Conde, S., Chaleteix, C., Pezet, D., and Balayssac, D. (2017). Long-Term Effects, Pathophysiological Mechanisms, and Risk Factors of Chemotherapy-Induced Peripheral Neuropathies: A Comprehensive Literature Review. Front. Pharmacol. 8:86. doi: 10.3389/fphar.2017.00086

Kono, T., Satomi, M., Suno, M., Kimura, N., Yamazaki, H., Furukawa, H., et al. (2012). Oxaliplatin-induced neurotoxicity involves TRPM8 in the mechanism of acute hypersensitivity to cold sensation. Brain Behav. 2, 68-73. doi: 10.1002/ brb3.34

Leonard, G. D., Wright, M. A., Quinn, M. G., Fioravanti, S., Harold, N., Schuler, B., et al. (2005). Survey of oxaliplatin-associated neurotoxicity using an interview-based questionnaire in patients with metastatic colorectal cancer. BMC Cancer 5:116. doi: 10.1186/1471-2407-5-116

Linden, D. J., Smeyne, M., and Connor, J. A. (1994). Trans-ACPD, a metabotropic receptor agonist, produces calcium mobilization and an inward current in cultured cerebellar Purkinje neurons. J. Neurophysiol. 71, 1992-1998. doi: 10. 1152/jn.1994.71.5.1992

Liu, N. K., Zhang, Y. P., Zou, J., Verhovshek, T., Chen, C., Lu, Q. B., et al. (2014). A semicircular controlled cortical impact produces long-term motor and cognitive dysfunction that correlates well with damage to both the sensorimotor cortex and hippocampus. Brain Res. 1576, 18-26. doi: 10.1016/j.brainres.2014. 05.042

Luo, Y., Suttle, A., Zhang, Q., Wang, P., and Chen, Y. (2021). Transient Receptor Potential (TRP) Ion Channels in Orofacial Pain. Mol. Neurobiol. [preprint]. doi: 10.1007/s12035-021-02284-2

Meents, J. E., Fischer, M. J., and McNaughton, P. A. (2017). Sensitization of TRPA1 by Protein Kinase A. PLoS One 12:e0170097. doi: 10.1371/journal.pone.0170097

Mizuno, K., Kono, T., Suzuki, Y., Miyagi, C., Omiya, Y., Miyano, K., et al. (2014). Goshajinkigan, a traditional Japanese medicine, prevents oxaliplatininduced acute peripheral neuropathy by suppressing functional alteration of TRP channels in rat. J. Pharmacol. Sci. 125, 91-98. doi: 10.1254/jphs.13 $244 \mathrm{fp}$

Molliver, D. C., Wright, D. E., Leitner, M. L., Parsadanian, A. S., Doster, K., Wen, D., et al. (1997). IB4-binding DRG neurons switch from NGF to GDNF dependence in early postnatal life. Neuron 19, 849-861. doi: 10.1016/s08966273(00)80966-6

Nakagawa, T., and Kaneko, S. (2017). Roles of Transient Receptor Potential Ankyrin 1 in Oxaliplatin-Induced Peripheral Neuropathy. Biol. Pharm. Bull. 40, 947-953. doi: 10.1248/bpb.b17-00243

Nassini, R., Gees, M., Harrison, S., De Siena, G., Materazzi, S., Moretto, N., et al. (2011). Oxaliplatin elicits mechanical and cold allodynia in rodents via TRPA1 receptor stimulation. Pain 152, 1621-1631. doi: 10.1016/j.pain.2011. 02.051

Nelson, M. T., Joksovic, P. M., Perez-Reyes, E., and Todorovic, S. M. (2005). The endogenous redox agent L-cysteine induces T-type Ca2+ channel-dependent sensitization of a novel subpopulation of rat peripheral nociceptors. J. Neurosci. 25, 8766-8775. doi: 10.1523/JNEUROSCI.2527-05.2005

Patil, M. J., Salas, M., Bialuhin, S., Boyd, J. T., Jeske, N. A., and Akopian, A. N. (2020). Sensitization of small-diameter sensory neurons is controlled by TRPV1 and TRPA1 association. FASEB J. 34, 287-302. doi: 10.1096/fj.201902026R

Rovini, A. (2019). Tubulin-VDAC Interaction: Molecular Basis for Mitochondrial Dysfunction in Chemotherapy-Induced Peripheral Neuropathy. Front. Physiol. 10:671. doi: 10.3389/fphys.2019.00671

Silverman, J. D., and Kruger, L. (1990). Selective neuronal glycoconjugate expression in sensory and autonomic ganglia: relation of lectin reactivity to peptide and enzyme markers. J. Neurocytol. 19, 789-801. doi: 10.1007/ BF01188046

Stucky, C. L., and Lewin, G. R. (1999). Isolectin B(4)-positive and -negative nociceptors are functionally distinct. J. Neurosci. 19, 6497-6505. doi: 10.1523/ jneurosci.19-15-06497.1999

Su, X., Wu, B., Zhang, W., Ji, Y. H., Wang, Q., and Tan, Z. Y. (2019) Inhibitory Effects of Columbianadin on Nociceptive Behaviors in a Neuropathic Pain Model, and on Voltage-Gated Calcium Currents in Dorsal Root Ganglion Neurons in Mice. Front. Pharmacol. 10:1522. doi: 10.3389/fphar.2019. 01522

Ta, L. E., Bieber, A. J., Carlton, S. M., Loprinzi, C. L., Low, P. A., and Windebank, A. J. (2010). Transient Receptor Potential Vanilloid 1 is essential for cisplatininduced heat hyperalgesia in mice. Mol. Pain 6:15. doi: 10.1186/1744-80 69-6-15

Tan, Z. Y., Lu, Y., Whiteis, C. A., Simms, A. E., Paton, J. F., Chapleau, M. W., et al. (2010). Chemoreceptor hypersensitivity, sympathetic excitation, and overexpression of ASIC and TASK channels before the onset of hypertension in SHR. Circ. Res. 106, 536-545. doi: 10.1161/CIRCRESAHA.109.206946

Trecarichi, A., and Flatters, S. J. L. (2019). Mitochondrial dysfunction in the pathogenesis of chemotherapy-induced peripheral neuropathy. Int. Rev. Neurobiol. 145, 83-126. doi: 10.1016/bs.irn.2019.05.001

Verge, V. M., Richardson, P. M., Benoit, R., and Riopelle, R. J. (1989). Histochemical characterization of sensory neurons with high-affinity receptors for nerve growth factor. J. Neurocytol. 18, 583-591. doi: 10.1007/BF01187079 
Viatchenko-Karpinski, V., Ling, J., and Gu, J. G. (2018). Down-regulation of Kv4.3 channels and a-type $\mathrm{K}(+)$ currents in V2 trigeminal ganglion neurons of rats following oxaliplatin treatment. Mol. Pain 14:1744806917750995. doi: 10.1177/ 1744806917750995

Vilceanu, D., Honore, P., Hogan, Q. H., and Stucky, C. L. (2010). Spinal nerve ligation in mouse upregulates TRPV1 heat function in injured IB4-positive nociceptors. J. Pain 11, 588-599. doi: 10.1016/j.jpain.2009.09.018

White, G., Lovinger, D. M., and Weight, F. F. (1989). Transient low-threshold $\mathrm{Ca} 2+$ current triggers burst firing through an afterdepolarizing potential in an adult mammalian neuron. Proc. Natl. Acad. Sci. U S A. 86, 6802-6806. doi: 10.1073/pnas.86.17.6802

Wu, B., McDermott, J. S., Krajewski, J. L., Knopp, K. L., Nisenbaum, E. S., Cummins, T. R., et al. (2019). Extracellular signal-regulated kinases mediate the enhancing effects of inflammatory mediators on resurgent currents in dorsal root ganglion neurons. Mol. Pain 15:1744806919837104. doi: 10.1177/ 1744806919837104

Wu, Z. Z., and Pan, H. L. (2004). Tetrodotoxin-sensitive and -resistant Na+ channel currents in subsets of small sensory neurons of rats. Brain Res. 1029, 251-258. doi: 10.1016/j.brainres.2004.09.051

Yamamoto, K., Chiba, N., Chiba, T., Kambe, T., Abe, K., Kawakami, K., et al. (2015). Transient receptor potential ankyrin 1 that is induced in dorsal root ganglion neurons contributes to acute cold hypersensitivity after oxaliplatin administration. Mol. Pain 11:69. doi: 10.1186/s12990-015-0072-8

Yue, C., and Yaari, Y. K. C. N. Q. (2004). /M channels control spike afterdepolarization and burst generation in hippocampal neurons. J. Neurosci. 24, 4614-4624. doi: 10.1523/JNEUROSCI.0765-04.2004

Zhang, X. L., Mok, L. P., Katz, E. J., and Gold, M. S. (2010). BKCa currents are enriched in a subpopulation of adult rat cutaneous nociceptive dorsal root ganglion neurons. Eur. J. Neurosci. 31, 450-462. doi: 10.1111/j.1460-9568.2009. 07060.x

Zhang, Y., Zhang, Y., Wang, Z., Sun, Y., Jiang, X., Xue, M., et al. (2021). Suppression of delayed rectifier $\mathrm{K}(+)$ channels by gentamicin induces membrane hyperexcitability through JNK and PKA signaling pathways in vestibular ganglion neurons. Biomed. Pharmacother. 135:111185. doi: 10.1016/ j.biopha.2020.111185

Zhao, M., Isami, K., Nakamura, S., Shirakawa, H., Nakagawa, T., and Kaneko, S. (2012). Acute cold hypersensitivity characteristically induced by oxaliplatin is caused by the enhanced responsiveness of TRPA1 in mice. Mol. Pain 8:55. doi: 10.1186/1744-8069-8-55

Zheng, J. H., Walters, E. T., and Song, X. J. (2007). Dissociation of dorsal root ganglion neurons induces hyperexcitability that is maintained by increased responsiveness to cAMP and cGMP. J. Neurophysiol. 97, 15-25. doi: 10.1152/ jn.00559.2006

Zheng, X., Tai, Y., He, D., Liu, B., Wang, C., Shao, X., et al. (2019). ETAR and protein kinase A pathway mediate ET-1 sensitization of TRPA1 channel: A molecular mechanism of ET-1-induced mechanical hyperalgesia. Mol. Pain 15:1744806919842473. doi: 10.1177/17448069198 42473

Zhou, J., Zhang, X., Zhou, Y., Wu, B., and Tan, Z. Y. (2019). Up-regulation of P2X7 Receptors Contributes to Spinal Microglial Activation and the Development of Pain Induced by BmK-I. Neurosci. Bull. 35, 624-636. doi: 10.1007/s12264-01900345-0

Zylka, M. J., Rice, F. L., and Anderson, D. J. (2005). Topographically distinct epidermal nociceptive circuits revealed by axonal tracers targeted to Mrgprd. Neuron 45, 17-25. doi: 10.1016/j.neuron.2004.12.015

Conflict of Interest: The authors declare that the research was conducted in the absence of any commercial or financial relationships that could be construed as a potential conflict of interest.

Copyright (c) $2021 \mathrm{Wu}$, Su, Zhang, Zhang, Feng, Ji and Tan. This is an open-access article distributed under the terms of the Creative Commons Attribution License (CC BY). The use, distribution or reproduction in other forums is permitted, provided the original author(s) and the copyright owner(s) are credited and that the original publication in this journal is cited, in accordance with accepted academic practice. No use, distribution or reproduction is permitted which does not comply with these terms. 